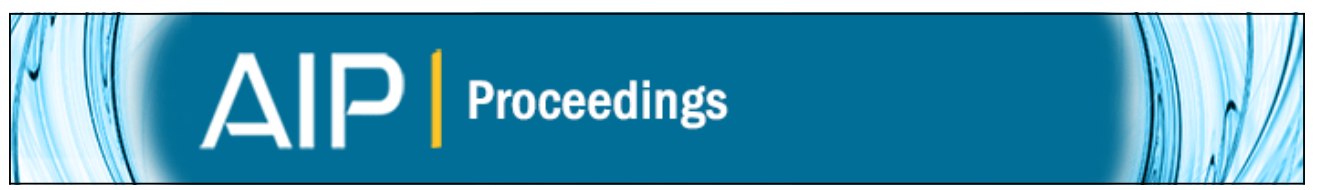

\title{
A Gravitational Redshift Determination of the Mean Mass of DBA White Dwarfs
}

Ross E. Falcon, D. E. Winget, M. H. Montgomery, and Kurtis A. Williams

Citation: AIP Conference Proceedings 1273, 13 (2010); doi: 10.1063/1.3527790

View online: http://dx.doi.org/10.1063/1.3527790

View Table of Contents:

http://scitation.aip.org/content/aip/proceeding/aipcp/1273?ver=pdfcov

Published by the AIP Publishing

\section{Articles you may be interested in}

Accretion and Diffusion in the DAZ White Dwarf GALEX J 1931+0117

AIP Conf. Proc. 1331, 246 (2011); 10.1063/1.3556207

The DBV White Dwarf EC20058-5234; the continuing story

AIP Conf. Proc. 1273, 542 (2010); 10.1063/1.3527884

Magnetic White Dwarfs in the SDSS and Estimating the Mean Mass of Normal DA and DB WDs

AIP Conf. Proc. 1273, 19 (2010); 10.1063/1.3527803

White Dwarfs in the HET Dark Energy Experiment

AIP Conf. Proc. 1273, 160 (2010); 10.1063/1.3527795

A Spitzer Warm Mission Proposal To Survey Single White Dwarfs

AIP Conf. Proc. 943, 248 (2007); 10.1063/1.2806784 


\title{
A Gravitational Redshift Determination of the Mean Mass of DBA White Dwarfs
}

\author{
Ross E. Falcon*, D. E. Winget*, M. H. Montgomery* and Kurtis A. \\ Williams $^{\dagger}$ \\ ${ }^{*}$ Department of Astronomy and McDonald Observatory, University of Texas, Austin, TX 78712, \\ USA \\ ${ }^{\dagger}$ Department of Physics and Astronomy, Texas A\&M University-Commerce, Commerce, TX 75428, \\ USA
}

\begin{abstract}
We measure apparent velocities ( $v_{\text {app }}$ ) of the $\mathrm{H} \alpha$ and $\mathrm{H} \beta$ Balmer line cores for 16 helium-dominated white dwarfs (WDs) using optical spectra taken for the European Southern Observatory SN Ia progenitor survey (SPY). Following the gravitational redshift method employed by Falcon et al. [1], we find a mean apparent velocity of $\left\langle v_{\text {app }}\right\rangle=39.58 \pm 4.41 \mathrm{~km} \mathrm{~s}^{-1}$ and use it to derive a mean mass of $\langle M\rangle=0.701_{-0.046}^{+0.042} M_{\odot}$. Though the sample is small, the mean mass appears to be larger than the mean mass of DAs derived using the same method $\left[0.647_{-0.014}^{+0.013} M_{\odot}, 1\right]$.
\end{abstract}

Keywords: white dwarfs, techniques: radial velocities, techniques: spectroscopic

PACS: $97.20 . \mathrm{Rp}, 95.85 . \mathrm{Kr}$

\section{INTRODUCTION}

White dwarfs (WDs) of spectral type DB constitute $\sim 20 \%$ of all WDs in the effective temperature range $30,000 \mathrm{~K} \gtrsim T_{\text {eff }} \gtrsim 10,000 \mathrm{~K}$ [2]. Their relatively small numbers combined with difficulties of interpreting pressure broadened helium lines $[3,4,5]$ contribute to our lack of understanding of their nature and origin.

Though helium is the dominant atmospheric constituent, Voss et al. [6] detect various amounts of hydrogen in most $(55 \%)$ of the DBs in their sample and find similar spectroscopic mass distributions to show that this WD subclass, DBAs, is not distinct from its parent class. Therefore, let us assume investigation of one is an investigation of both. We do not discard the possibility, however, that DBAs are fundamentally different from DBs and possess signficantly different masses.

We follow the method of Falcon et al. [1], which uses the apparent velocities of hydrogen Balmer lines for an ensemble of WDs to determine a mean gravitational redshift. With a mean redshift, we derive a mean mass.

\section{OBSERVATIONS}

We use spectroscopic data from the European Southern Observatory (ESO) SN Ia progenitor survey [SPY; 7]. These observations, taken using the UV-Visual Echelle Spectrograph [UVES; 8] at Kueyen, Unit Telescope 2 of the ESO VLT array, constitute the largest, homogeneous, high-resolution $\left(0.36 \AA\right.$ or $\sim 16 \mathrm{~km} \mathrm{~s}^{-1}$ at $\left.\mathrm{H} \alpha\right)$ spectroscopic 
dataset for WDs. We obtain the pipeline-reduced data online through the publicly available ESO Science Archive Facility.

\subsection{Sample}

We analyze 16 helium-dominated WDs from the SPY sample that show measurable apparent velocity $\left(v_{\text {app }}\right.$ ) in the $\mathrm{H} \alpha$ (and $\mathrm{H} \beta$ ) line cores. Since these DBAs do not show measurable $v_{\text {app }}$ variations, we presume them to be non-binary.

We require our targets to have $T_{\text {eff }}>16,500 \mathrm{~K}$ (see Section 3.2). The lack of detectable Zeeman splitting implies that our targets do not harbor significant ( $\gtrsim 100 \mathrm{kG}$ ) magnetic fields [e.g., 9].

The literature does not show our WDs to have any stringent stellar population classifications; none are suspected to belong specifically to the thick disk or halo. Because it is a necessary criterion for our analysis (see Section 3.3), we make the assumption that all of our targets are from the thin disk. Nearly all (>90\%) of the SPY WDs that have been studied kinematically are classified as thin disk objects [10, 11].

\section{USING GRAVITATIONAL REDSHIFT TO DETERMINE A MEAN MASS}

We summarize the method employed in Falcon et al. [1]:

\subsection{Gravitational Redshift}

In the weak-field limit, the general relativistic effect of gravitational redshift can be observed as a velocity shift in absorption lines and is expressed as

$$
v_{g}=\frac{c \Delta \lambda}{\lambda}=\frac{G M}{R c}
$$

where $G$ is the gravitational constant, and $c$ is the speed of light. In our case, $M$ is the WD mass, and $R$ is the WD radius.

The apparent velocity of an absorption line is the sum of this gravitational redshift and the stellar radial velocity: $v_{\mathrm{app}}=v_{\mathrm{g}}+v_{\mathrm{r}}$. These two components cannot be explicitly separated for individual WDs without an independent $v_{\mathrm{r}}$ measurement or mass determination. We break this degeneracy for a group of WDs by assuming that the sample is co-moving and local. After we correct each $v_{\text {app }}$ to the local standard of rest (LSR), only random stellar motions dominate the dynamics of our sample. These average out, and the mean apparent velocity becomes the mean gravitational redshift: $\left\langle v_{\mathrm{app}}\right\rangle=\left\langle v_{\mathrm{g}}\right\rangle$. 


\subsection{Velocity Measurements}

Collisional (Stark) broadening effects cause asymmetry in the wings of absorption lines for the hydrogen Balmer series, making it difficult to measure a velocity centroid $[12,13]$. These effects are not significant in the sharp, non-LTE $\mathrm{H} \alpha$ and $\mathrm{H} \beta$ Balmer line cores.

van der Waals broadening due to neutral He may also signficantly affect line shapes below $T_{\text {eff }}>16,500 \mathrm{~K}[14,6]$. We avoid this systematic by not including targets in that range of $T_{\text {eff }}$.

We measure $v_{\text {app }}$ for each target in our sample by fitting a Gaussian profile to the $\mathrm{H} \alpha$ line core using GAUSSFIT, a non-linear least-squares fitting routine in IDL. When available, we combine this measurement with that of the $\mathrm{H} \beta$ line core centroid as a mean weighted according to the uncertainties returned by the fitting routine. We include $\mathrm{H} \beta$ line core centroid information in 4 of our $16 v_{\text {app }}$ measurements. If multiple epochs of observations exist, we combine these measurements as a weighted mean as well. $v_{\text {app }}$ measurements of a given observation (i.e., $\mathrm{H} \alpha$ and $\mathrm{H} \beta$ line core centroids) are combined before multiple epochs.

\subsection{Co-Moving Approximation}

We measure a mean gravitational redshift by assuming that our WDs are a co-moving, local sample. With this assumption, only random stellar motions dominate the dynamics of our targets; this falls out when we average over the sample.

For this assumption to be valid, at least as an approximation, our WDs must belong to the same kinematic population; in the case of this work, this is the thin disk. We achieve a co-moving group by correcting each measured $v_{\text {app }}$ to the kinematical LSR described by Standard Solar Motion [15]. In Falcon et al. [1], we show in detail that this is a suitable choice of reference frame for the purpose of this investigation.

Our WDs must also reside at distances that are small when compared to the size of the Galaxy, thereby making systematics introduced by the Galactic kinematic structure negligible. Six of our targets have published distance determinations in the range of $\sim 40$ to $\sim 260 \mathrm{pc}[16,17,18]$. We assume the rest to have comparable distances to the rest of the SPY sample which Pauli et al. [10] determined spectroscopically to be mostly ( $\gtrsim 90 \%$ ) within $200 \mathrm{pc}$ with a mean of $\sim 100 \mathrm{pc}$.

\subsection{Deriving a Mean Mass}

To translate the mean apparent velocity $\left\langle v_{\text {app }}\right\rangle$ to a mean mass, we invoke two dependencies: (1) we need the mass-radius relation from an evolutionary model, and (2) since the WD radius does slightly contract during its cooling sequence, we need an estimate of the position along this track for the average WD in our sample (i.e., a mean $T_{\text {eff }}$ ).

Our evolutionary models use $M_{\mathrm{He}} / M_{\star}=10^{-2}$ helium surface-layer mass, and, since we are interested in WDs with helium-dominated atmospheres, we use $M_{\mathrm{H}} / M_{\star}=0$ 
TABLE 1. Mean Apparent Velocities

\begin{tabular}{cccccccc}
\hline Sample & \# of WDs & $\begin{array}{c}\left\langle v_{\text {app }}\right\rangle \\
\left(\mathrm{km} \mathrm{s}^{-1}\right)\end{array}$ & $\begin{array}{c}\delta\left\langle v_{\text {app }}\right\rangle \\
\left(\mathrm{km} \mathrm{s}^{-1}\right)\end{array}$ & $\begin{array}{c}\sigma_{v_{\text {app }}} \\
\left(\mathrm{km} \mathrm{s}^{-1}\right)\end{array}$ & $\begin{array}{c}\left\langle\delta v_{\text {app }}\right\rangle \\
\left(\mathrm{km} \mathrm{s}^{-1}\right)\end{array}$ & $\begin{array}{c}\langle M / R\rangle \\
\left(\mathrm{M}_{\odot} / \mathrm{R}_{\odot}\right)\end{array}$ & $\begin{array}{c}\delta\langle M / R\rangle \\
\left(\mathrm{M}_{\odot} / \mathrm{R}_{\odot}\right)\end{array}$ \\
\hline DBA & 16 & 39.58 & 4.41 & 16.77 & 4.42 & 62.20 & 6.95 \\
DA $^{*}$ & 449 & 32.57 & 1.17 & 24.84 & 1.51 & 51.19 & 1.84 \\
\hline
\end{tabular}

* Main sample of normal DAs from Falcon et al. [1]

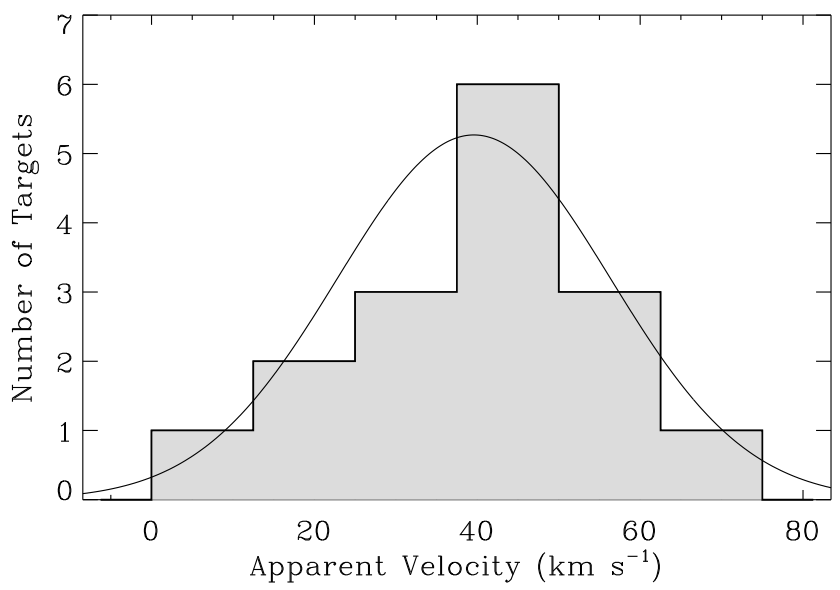

FIGURE 1. Histogram of measured apparent velocities $v_{\text {app }}$ with a bin size of $12.5 \mathrm{~km} \mathrm{~s}^{-1}$. The mean $v_{\text {app }}$ is $39.58 \pm 4.41 \mathrm{~km} \mathrm{~s}^{-1}$. The overplotted curve is the Gaussian distribution function used to determine Monte Carlo uncertainties.

for the hydrogen surface-layer mass. See Montgomery et al. [19] for a more complete description of our models. Our dependency on evolutionary models is small. We are interested in the mass-radius relation from these models, and this is relatively straightforward since WDs are mainly supported by electron degeneracy pressure, making the WD radius a weak function of temperature. We estimate that varying the $\mathrm{C} / \mathrm{O}$ ratio in the core affects the radius by less than $0.7 \%$.

\section{RESULTS}

We observe $\mathrm{H}$ absorption in 16 WDs classifed as helium-dominated by Voss et al. [6]. Table 1 lists our derived mean apparent velocity as well as the corresponding result for DAs from Falcon et al. [1]. Figure 1 shows the $v_{\text {app }}$ distribution.

The quoted uncertainty of the mean apparent velocity (Column 4 of Table 1) comes from Monte Carlo simulations. For the sample, we recreate a large number of instances $(10,000)$ of the $v_{\text {app }}$ distribution by randomly sampling from a convolution of the empir- 


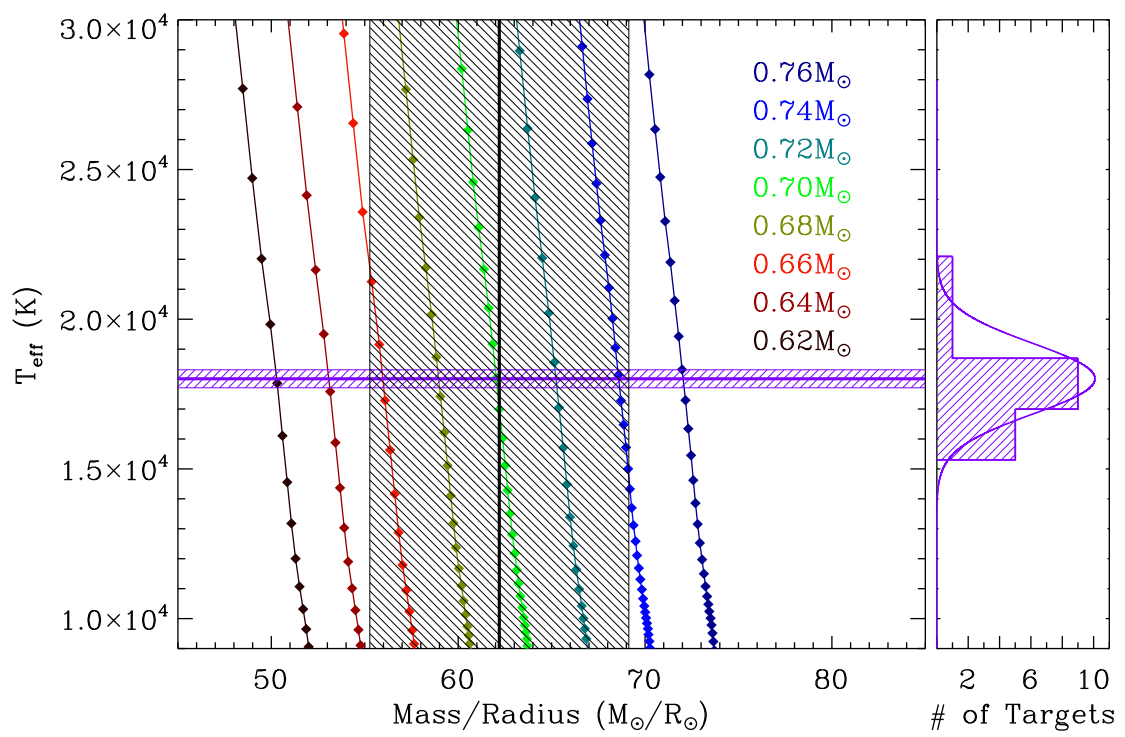

FIGURE 2. Left: plot of $M / R$ versus $T_{\text {eff }}$ with cooling tracks from evolutionary models for a range of WD masses. The intersection of the mean measured apparent velocity $v_{\text {app }}$ (vertical, black line) and mean $T_{\text {eff }}$ from (horizontal, purple line) indicates a mean mass of $0.701_{+0.046}^{+0.042} \mathrm{M}_{\odot}$. Right: Distribution of spectroscopically determined $T_{\text {eff }}$ of our targets from Voss et al. [6]. The bin size is $1700 \mathrm{~K}$. The mean is $18,010 \pm 300 \mathrm{~K}$. The overplotted curve is the empirical distribution function used to determine Monte Carlo uncertainties.

ical $v_{\text {app }}$ distribution (Gaussian characterized by the parameters in Columns 3 and 5 of Table 1) and the empirical measurement uncertainty distribution (not shown). We adopt the standard deviation of the resulting simulated mean values as our formal uncertainty. Since the input distribution for our simulations is empirical, our uncertainty is subject to the normal limitations of Frequentist statistics.

For this sample, $\left\langle v_{\text {app }}\right\rangle=39.58 \pm 4.41 \mathrm{~km} \mathrm{~s}^{-1}$. Using spectroscopically determined $T_{\text {eff }}$ from Voss et al. [6], $\left\langle T_{\text {eff }}\right\rangle=18,010 \pm 300 \mathrm{~K}$. We plot these values against WD cooling tracks from our evolutionary models in Figure 2 and interpolate to find $\langle M\rangle=$ $0.701_{-0.046}^{+0.042} M_{\odot}$. This is larger than the mean mass for normal DAs determined using the same gravitational redshift method $\left[0.647 M_{\odot} ; 1\right]$. This also disagrees with $0.621 M_{\odot}$, the mean of the spectroscopic mass determinations for these targets from Voss et al. [6].

\section{ACKNOWLEDGMENTS}

R.E.F. thanks the SPY Collaboration for providing reduced spectra of excellent quality. The observations were made with the European Southern Observatory telescopes and obtained from the ESO/ST-ECF Science Archive Facility. This work has made use of 
NASA's Astrophysics Data System Bibliographic Services. It has also made use of the SIMBAD database, operated at CDS, Strasbourg, France. This work is supported by the National Science Foundation under grant AST-0909107, the Norman Hackerman Advanced Research Program under grant 003658-0255-2007, and the Institute for High Energy Density Science, funded by The University of Texas System and supported in part by Sandia National Laboratories. M.H.M acknowledges the support of the Delaware Asteroseismic Research Center.

\section{REFERENCES}

1. R. E. Falcon, D. E. Winget, M. H. Montgomery, and K. A. Williams, ApJ, 712, 585-595 (2010).

2. A. Beauchamp, F. Wesemael, P. Bergeron, J. Liebert, and R. A. Saffer, "The DB and DBA white dwarfs: epitomes of hydrogen-deficient stars," in Hydrogen Deficient Stars, edited by C. S. Jeffery \& U. Heber, 1996, vol. 96 of Astronomical Society of the Pacific Conference Series, pp. 295-+.

3. A. Beauchamp, F. Wesemael, and P. Bergeron, ApJS, 108, 559-+ (1997).

4. A. Beauchamp, and F. Wesemael, ApJ, 496, 395-+ (1998).

5. A. Beauchamp, F. Wesemael, P. Bergeron, G. Fontaine, R. A. Saffer, J. Liebert, and P. Brassard, ApJ, 516, 887-891 (1999).

6. B. Voss, D. Koester, R. Napiwotzki, N. Christlieb, and D. Reimers, A\&A, 470, 1079-1088 (2007).

7. R. Napiwotzki, N. Christlieb, H. Drechsel, H.-J. Hagen, U. Heber, D. Homeier, C. Karl, D. Koester, B. Leibundgut, T. R. Marsh, S. Moehler, G. Nelemans, E.-M. Pauli, D. Reimers, A. Renzini, and L. Yungelson, Astronomische Nachrichten, 322,411-418 (2001), arXiv: astro-ph/ 0203147.

8. H. Dekker, S. D'Odorico, A. Kaufer, B. Delabre, and H. Kotzlowski, "Design, construction, and performance of UVES, the echelle spectrograph for the UT2 Kueyen Telescope at the ESO Paranal Observatory," in Society of Photo-Optical Instrumentation Engineers (SPIE) Conference Series, edited by M. Iye, and A. F. Moorwood, 2000, vol. 4008 of Society of Photo-Optical Instrumentation Engineers (SPIE) Conference Series, pp. 534-545.

9. D. Koester, B. Voss, R. Napiwotzki, N. Christlieb, D. Homeier, T. Lisker, D. Reimers, and U. Heber, $A \& A, \mathbf{5 0 5}, 441-462$ (2009).

10. E.-M. Pauli, R. Napiwotzki, U. Heber, M. Altmann, and M. Odenkirchen, A\&A, 447, 173-184 (2006).

11. R. Richter, U. Heber, and R. Napiwotzki, "3D-Kinematics of White Dwarfs from the SPY-Project," in 15th European Workshop on White Dwarfs, edited by R. Napiwotzki, and M. R. Burleigh, 2007, vol. 372 of Astronomical Society of the Pacific Conference Series, pp. 107-+.

12. H. L. Shipman, and R. G. Mehan, ApJ, 209, 205-207 (1976).

13. B. Grabowski, J. Halenka, and J. Madej, ApJ, 313, 750-756 (1987).

14. D. Koester, R. Napiwotzki, B. Voss, D. Homeier, and D. Reimers, A\&A, 439, 317-321 (2005).

15. F. J. Kerr, and D. Lynden-Bell, MNRAS, 221, 1023-1038 (1986).

16. J. Farihi, E. E. Becklin, and B. Zuckerman, ApJS, 161, 394-428 (2005).

17. B. G. Castanheira, S. O. Kepler, G. Handler, and D. Koester, A\&A, 450, 331-337 (2006).

18. M. Limoges, and P. Bergeron, ApJ, 714, 1037-1051 (2010).1003 . 4313.

19. M. H. Montgomery, E. W. Klumpe, D. E. Winget, and M. A. Wood, ApJ, 525, 482-491 (1999). 